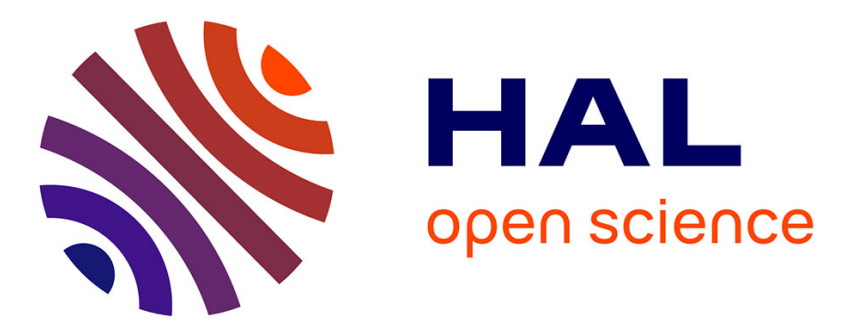

\title{
Consommation et surconsommation de cannabis : apports et limites de l'épidémiologie
}

François G. E. Beck, Stéphane Legleye, Stanislas Spilka

\section{To cite this version:}

François G. E. Beck, Stéphane Legleye, Stanislas Spilka. Consommation et surconsommation de cannabis: apports et limites de l'épidémiologie. Psychotropes, 2007, 13 (1), pp.9-32. 10.3917/psyt.131.0009 . halshs-00285202

\section{HAL Id: halshs-00285202 \\ https://shs.hal.science/halshs-00285202}

Submitted on 4 Jun 2008

HAL is a multi-disciplinary open access archive for the deposit and dissemination of scientific research documents, whether they are published or not. The documents may come from teaching and research institutions in France or abroad, or from public or private research centers.
L'archive ouverte pluridisciplinaire HAL, est destinée au dépôt et à la diffusion de documents scientifiques de niveau recherche, publiés ou non, émanant des établissements d'enseignement et de recherche français ou étrangers, des laboratoires publics ou privés. 


\title{
Consommation et surconsommation de cannabis : apports et limites de l'épidémiologie
}

\author{
Cannabis use and misuse : \\ findings and limits of epidemiology
}

\author{
François Beck ${ }^{1,2}$, Stéphane Legleye ${ }^{3,4}$, Stanislas Spilka ${ }^{3}$ \\ François Beck \\ Responsable du Département des Sciences Humaines \\ Institut national de prévention et d'éducation pour la santé (Inpes) \\ 42 bd de la Libération - F 93203 Saint-Denis Cedex \\ Courriel : francois.beck@inpes.sante.fr
}

Résumé : L'usage de cannabis apparait présent dans tous les segments de la population. Le genre, l'âge, le niveau d'instruction scolaire, l'activité professionnelle et la zone de résidence sont les principaux facteurs discriminants. Ainsi, il s'avère plus fréquent parmi les hommes et les jeunes générations, mais aussi, par exemple, parmi les chômeurs, tandis que les différences entre les actifs occupés se révèlent plus faibles. De même, l'expérimentation et les usages occasionnels se révèlent plus répandus parmi les personnes diplômées du supérieur, au contraire des usages plus réguliers. Ces déterminants sociaux permettent de décrire et de rendre compte des usages dans la population, mais ne peuvent suffire à les expliquer; ils masquent en effet partiellement les opportunités, les choix et les préférences individuelles de consommation de produits psychoactifs et occultent en particulier que les usages sont très nettement liés à une sociabilité et à un mode de vie. Comme le soulignent les usagers lorsqu'ils énoncent leurs motifs de consommation et d'abandon, le cannabis est partie intégrante de la vie de nombreuses personnes qui ne semblent pas en éprouver de difficultés majeures. Dans l'étude de la surconsommation, il convient d'isoler au sein des usages réguliers, ceux qui seraient problématiques. Si l'épidémiologie de la surconsommation de cannabis reste parcellaire, elle a déjà donné quelques résultats qui rejoignent la pratique des acteurs de terrain, tel que le lien fort entre une telle pratique et une entrée précoce dans les usages. Les véritables avancées sont toutefois à venir, lorsque les instruments de mesure de l'usage problématique de cannabis auront été validés tant au niveau épidémiologique qu'en clinique et utilisés dans les enquêtes en population générale.

Abstract: Cannabis use is widespread in every parts of the French population. Gender, age, school achievement, employement and place of living are the most prominent associated social and demographic factors. On the one hand, levels of use are higher among men, youngsters and unemployed whereas differences

\footnotetext{
${ }^{1}$ Institut national de prévention et d'éducation pour la santé (Inpes).

${ }^{2}$ Cesames : Centre de recherche psychotropes, santé mentale, société (CNRS UMR 8136, Inserm U611, université René Descartes Paris V).

${ }^{3}$ Observatoire français des drogues et des toxicomanies (OFDT).

${ }^{4}$ Inserm
} 
according to type of occupation are rather small; on the other hand, people with high levels of diploma seem to be less frequently regular users than people with poorer level of education. These social factors hide some other aspects of cannabis use, such as lifestyle, sociability and leisure, or personal choices. Most of the cannabis users don't seem to have problems with their use. In the study of regular use, it reveals crucial to distinguish those of the regular users who are in a problematic situation. However, the conception of screening tests for problematic use has been a recent and challenging public health concern. But defining a threshold for effective or future problematic use is a difficult task. The second part of the article describes some of the general aspects of this work and presents some results for France, and their limits. Improvements in the epidemiology of cannabis misuse are to come with the clinical validations of the cannabis screening tests.

Mots clés : cannabis, épidémiologie, surconsommation, adolescence, usage problématique.

Keywords: cannabis, epidemiology, overconsumption, adolescence, problematic use.

\section{Introduction}

Le cannabis a longtemps fait l'objet de débats au cours desquels les éléments passionnels l'emportaient sur un énoncé plus objectif des faits. Sur l'ampleur des consommations au sein de la population, et en particulier des consommations les plus intenses, les chiffres les plus fantaisistes ont souvent circulé (Beck, 2006). Ce n'était pas tant faute de volonté que de moyens : la consommation de cannabis en France n'est en fait observée que depuis une quinzaine d'années grâce aux enquêtes représentatives en population générale.

Cette observation s'appuie, en particulier, sur les Baromètres santé coordonnés par l'Inpes depuis le début des années 1990 ainsi que sur l'Enquête sur la santé et les consommations lors de l'appel de préparation à la défense (Escapad), mise en place par l'OFDT depuis l'année 2000.

Les derniers exercices de ces enquêtes ont été réalisés en 2005 et constituent à ce jour les données les plus récentes. Ils permettent une description détaillée des consommations et des consommateurs, ainsi que la documentation de quelques questions sur la nature des usages observés : existe-t-il une consommation type de cannabis ? Y a-t-il une norme au-delà de laquelle la consommation serait surconsommation, usage nocif ou délétère?

La première partie de ce travail rappelle les principales données épidémiologiques décrivant la diffusion de la substance dans la population et brosse un portrait sommaire des usagers.

La seconde s'interroge sur les conditions, les limites et la pertinence de la mesure de la surconsommation de cannabis par le biais des enquêtes en population générale. 


\section{Repères méthodologiques}

Depuis le début des années 1990, l'Institut national de prévention et d'éducation pour la Santé (Inpes) mène, en partenariat avec de nombreux acteurs de santé, une série d'enquêtes intitulées Baromètre santé, qui abordent les différents comportements et attitudes de santé des Français. Ces enquêtes sont réalisées à l'aide du système de Collecte assistée par téléphone et informatique (Cati). L'anonymat et le respect de la confidentialité sont garantis par une procédure d'effacement du numéro de téléphone ayant reçu l'aval de la Commission nationale informatique et liberté (Cnil).

En 2005, pour faire face à l'abandon récent du téléphone filaire au profit du mobile, un échantillon d'environ 4000 individus issus de ménages ne possédant qu'un téléphone mobile a été interrogé en plus des 26500 individus possédant une ligne fixe à leur domicile. La passation du questionnaire durait en moyenne quarante minutes pour les personnes interrogées sur téléphone fixe et un peu plus d'un quart d'heure pour les personnes interrogées sur téléphone portable. L'échantillon comprend finalement 30514 individus de 12 à 75 ans.

L'enquête Escapad se déroule, grâce à une collaboration avec la Direction du service national (DSN), lors de la Journée d'appel de préparation à la défense (la $\mathrm{JAPD}$ ). Une fois par an, dans toute la France, les jeunes qui participent à cette journée répondent à un questionnaire auto-administré anonyme centré sur leurs consommations de substances psychoactives. Ces adolescents, majoritairement âgés de 17 ans sont de nationalité française et pour une grande part, encore scolarisés dans l'enseignement secondaire, mais certains d'entre eux sont actifs, en apprentissage ou en études supérieures. En 2005, 37512 individus ont été interrogés, dont 32057 en métropole, âgés de 16 à 23 ans, mais très majoritairement âgés de 17 ans. Après contrôle de la qualité des données et filtrage sur l'âge, on dénombre 29393 questionnaires exploitables en métropole.

\section{Données épidémiologiques et portraits des usagers}

\section{Le cannabis, première drogue illicite}

Le cannabis est de loin la drogue illicite la plus consommée et la plus disponible en France. Parmi les 15 à 64 ans, trois individus sur dix $(30,6 \%)^{5}$ l'ont déjà expérimenté tandis que $43,1 \%$ disent s'en être déjà vu proposer. À titre de comparaison, viennent ensuite, dans des proportions nettement moindres, l'expérimentation du poppers $(3,9 \%)$, des champignons hallucinogènes $(2,7 \%)$, de la cocaïne $(2,6 \%)$ et de l'ecstasy $(2,0 \%)$. Moins de $2 \%$ ont déclaré avoir expérimenté des produits à inhaler, du LSD, des amphétamines, et moins de $1 \%$ de l'héroïne ou du crack. Près de $9 \%$ déclarent avoir consommé du cannabis au cours des douze derniers mois, tandis que l'usage actuel des autres drogues illicites concerne moins de $1 \%$ des individus.

Ces pourcentages peuvent être ramenés à des effectifs de la population qui permettent de porter un regard complémentaire sur la diffusion des usages. Le cannabis totalise ainsi plus de douze millions d'expérimentateurs parmi les 12-75 ans, loin devant la cocaïne et l'ecstasy (près d'un million) et l'héroïne, qui en

\footnotetext{
${ }^{5}$ Dont $3,1 \%$ déclarant « non » à la question de l'usage au cours de la vie, mais précisant ultérieurement avoir déjà consommé « pour y goûter ».
} 
compte près de quatre fois moins. Globalement, les produits licites, dont l'alcool et le tabac, sont nettement plus répandus, qu'il s'agisse de l'expérimentation, de l'usage actuel ou des usages plus réguliers. L'usage régulier de cannabis concerne plus d'un million de personnes et son usage quotidien plus d'un demi million (Beck, et al., 2006a).

Tableau 1 : Niveaux d'usage et de proposition par produits parmi les 15-64 ans

\begin{tabular}{|l|c|c|c|}
\hline & Expérimentation & Usage actuel & Proposition reçue \\
\hline Cannabis & $30,6 \%$ & $8,6 \%$ & $43,1 \%$ \\
Poppers & $3,9 \%$ & $0,6 \%$ & $8,2 \%$ \\
Champignons & $2,7 \%$ & $0,3 \%$ & $7,0 \%$ \\
hallucinogènes & $2,6 \%$ & $0,6 \%$ & $8,0 \%$ \\
Cocaïne & $2,0 \%$ & $0,5 \%$ & $8,9 \%$ \\
Ecstasy & $1,7 \%$ & $0,2 \%$ & $4,8 \%$ \\
Produits à inhaler & $1,5 \%$ & $0,1 \%$ & $4,6 \%$ \\
LSD & $1,4 \%$ & $0,2 \%$ & $5,2 \%$ \\
Amphétamines & $0,8 \%$ & $0,1 \%$ & $4,6 \%$ \\
Héroïne & $0,3 \%$ & $0,1 \%$ & $2,9 \%$ \\
Crack & \multicolumn{2}{|c|}{. } & \\
\hline
\end{tabular}

Source : Baromètre santé 2005, Inpes, exploitation OFDT

Tableau 2 : Estimation du nombre de consommateurs de substances psychoactives en France métropolitaine parmi les $12-75$ ans en $2005^{\circ}$

\begin{tabular}{|l|c|c|c|c|c|c|}
\hline & Alcool & Tabac & Cannabis & Héroïne & Cocaïne & Ecstasy \\
\hline Expérimentateurs & $42,5 \mathrm{M}$ & $34,8 \mathrm{M}$ & $12,4 \mathrm{M}$ & 350000 & $1,1 \mathrm{M}$ & 900000 \\
- dont actuels & $39,4 \mathrm{M}$ & $14,9 \mathrm{M}$ & $3,9 \mathrm{M}$ & $/ /$ & 250000 & 200000 \\
- dont réguliers & $9,7 \mathrm{M}$ & $11,8 \mathrm{M}$ & $1,2 \mathrm{M}$ & $/ /$ & $/ /$ & $/ /$ \\
- dont quotidiens & $6,4 \mathrm{M}$ & $11,8 \mathrm{M}$ & 550000 & $/ /$ & $/ /$ & $/ /$ \\
\hline
\end{tabular}

Sources : Escapad 2003, OFDT ; Espad 2003, Inserm/OFDT/MJENR ; Baromètre santé 2005, Inpes, exploitation OFDT

- $\quad / /$ : non disponible

- Expérimenteur : au moins un usage au cours de la vie (cet indicateur sert principalement à mesurer la diffusion d'un produit dans la population);

- Usage actuel : au moins un usage dans l'année (pour le tabac, il s'agit des personnes déclarant fumer ne serait-ce que de temps en temps);

- Usage régulier : au moins trois consommations d'alcool dans la semaine, tabagisme quotidien, 10 consommations de cannabis dans le mois.

\section{D'importants clivages par sexe et âge}

Comme pour l'alcool et les autres drogues illicites, les hommes se déclarent proportionnellement nettement plus souvent consommateurs de cannabis que les femmes, la différence étant d'autant plus importante que l'on observe des niveaux d'usage élevés. Le détail montre en effet que l'usage au cours du mois concerne

\footnotetext{
${ }^{6}$ Le nombre d'individus de 12-75 ans en 2005 est d'environ 46 millions. Les chiffres de ce tableau sont des ordres de grandeur et doivent de ce fait être lus comme des données de cadrage. En effet, une marge d'erreur existe, même si elle s'avère raisonnable. Par exemple, la mention des 12,4 millions d'expérimentateurs de cannabis signifie que le nombre d'expérimentateurs se situe vraisemblablement entre 12,2 et 12,6 millions.
} 
4,8 $\%$ des $15-64$ ans (7,3\% des hommes et 2,5\% des femmes), l'usage régulier $2,8 \%(4,3 \%$ vs $1,3 \%)$ et enfin l'usage quotidien $1,3 \%(2,0 \%$ vs $0,5 \%)$.

Les usages se raréfient très vite avec l'âge des personnes (figure 1), montrant que si le cannabis est un produit « jeune » parce qu'il se diffuse parmi les générations les moins âgées, son usage est le plus souvent, jusqu'à ces dernières années, abandonné lors du passage à la vie adulte.

Figure 1 : usages de cannabis au cours de la vie et de l'année, par sexe et âge

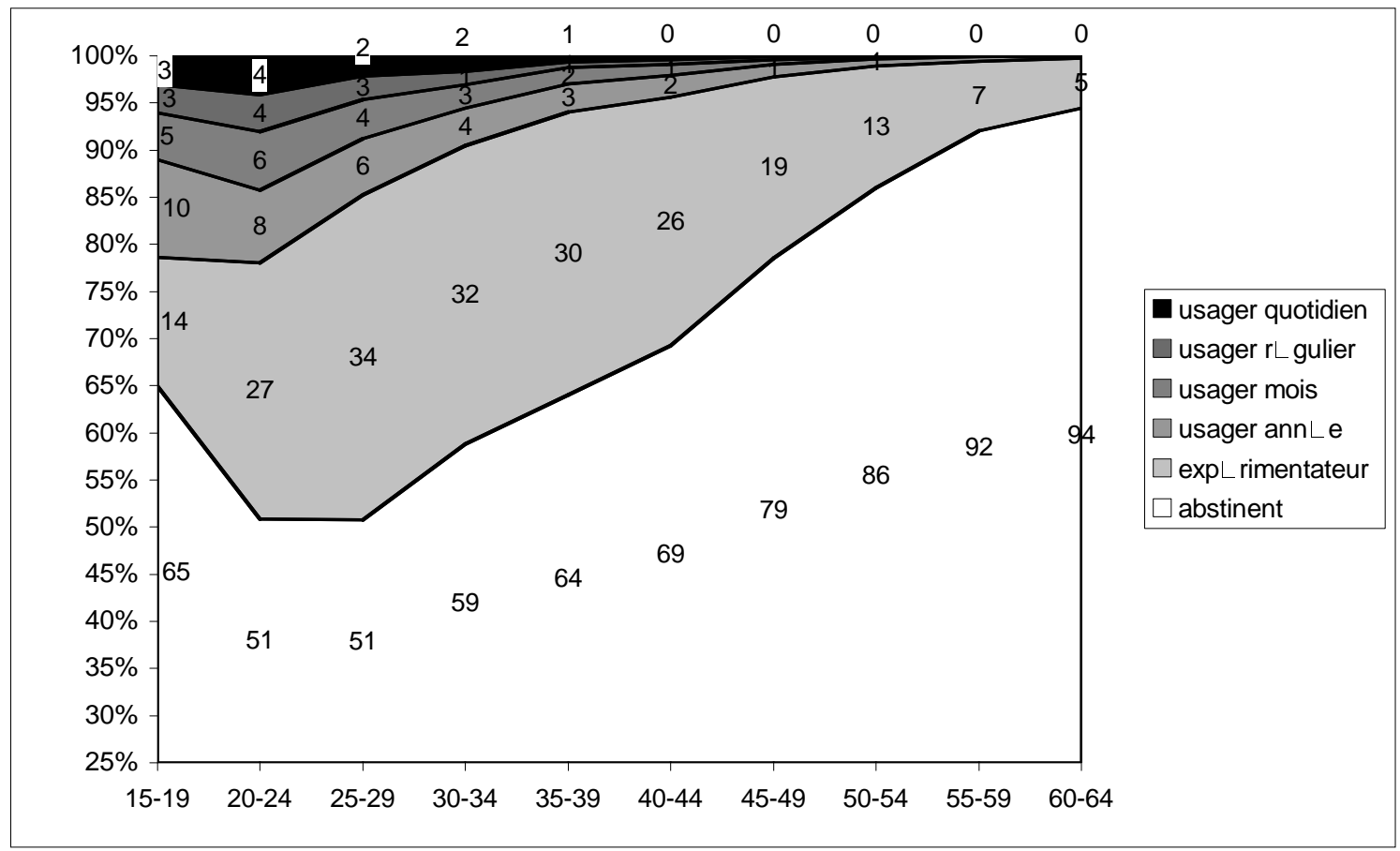

Note : l'axe des ordonnées est tronqué à $25 \%$.

Source : Baromètre santé 2005, Inpes, exploitation OFDT

Ces disparités illustrent le fait que le consommateur type de cannabis est un homme, plutôt jeune. Une analyse plus détaillée montre que les usages au cours des douze derniers mois sont majoritairement concentrés ( $80 \%$ d'entre eux environ) entre 15 et 34 ans. Les consommateurs plus âgés apparaissent ainsi relativement atypiques, et ce d'autant plus qu'ils sont usagers plus fréquents : près de $90 \%$ des usagers réguliers ont entre 15 et 34 ans.

\section{Des disparités importantes suivant le statut d'activité et le niveau d'étude}

Les usages semblent se répartir inégalement suivant le statut scolaire et professionnel des personnes interrogées. Ils apparaissent particulièrement faibles parmi les inactifs, catégorie composée majoritairement de personnes au foyer, tandis que c'est parmi les élèves et étudiants qu'ils se trouvent les plus répandus. Les niveaux de consommation des chômeurs s'avèrent relativement proches de ceux des élèves et étudiants pour l'expérimentation, mais nettement moins élevés pour les usages fréquents. Parmi les actifs occupés, les écarts s'avèrent plus faibles, mais il est possible de distinguer grossièrement trois groupes de professions et catégories sociales (PCS) : les agriculteurs exploitants d'un côté, largement sous-consommateurs, les artisans, commerçants et chefs d'entreprise, ainsi que les ouvriers de l'autre, nettement sur-consommateurs, tandis que les cadres et professions intermédiaires présentent une consommation proche de la moyenne. L'observation des figures 1 et 2 suggère ainsi que la consommation de 
cannabis est abandonnée par une majorité d'individus au moment de l'entrée dans le monde du travail, mais que le chômage peut constituer une circonstance de relatif maintien dans cette pratique.

Figure 2 : usages de cannabis suivant le statut scolaire et professionnel parmi les $15-34$ ans (\%)

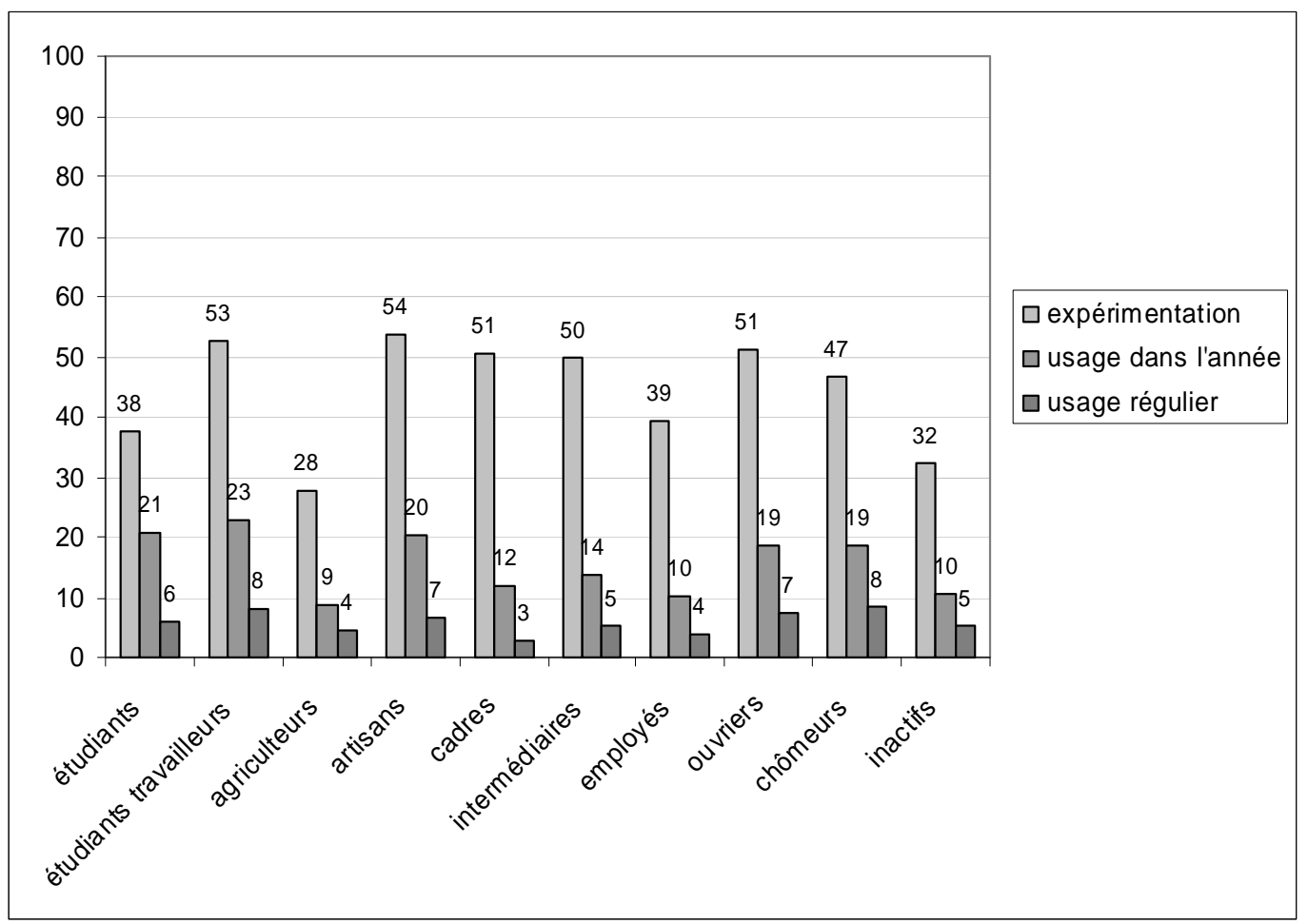

Source : Baromètre santé 2005, Inpes, exploitation OFDT

Ces résultats sont toutefois descriptifs et n'autorisent pas de réelle comparaison des catégories représentées. En effet, celles-ci diffèrent très largement par l'âge moyen, la féminisation, la situation familiale, etc. Par exemple, les étudiants sont en moyenne plus jeunes que les inactifs et présentent de ce fait des niveaux d'usage supérieurs, et de semblables distinctions peuvent être faites au sein des différentes catégories d'actifs occupés.

Comparer ainsi les catégories revient à vérifier s'il persiste un effet de l'appartenance à une catégorie donnée qui ne soit pas réductible au sexe, à l'âge, etc. Pour cela, il convient de corriger les facteurs de confusion et raisonner « toutes choses égales par ailleurs » dans des analyses multivariées.

Ainsi, dans une régression logistique, les élèves et étudiants apparaissent toutes choses égales par ailleurs légèrement sous-expérimentateurs en comparaison des actifs occupés, mais leurs usages plus récents ne se distinguent pas. Les étudiants exerçant par ailleurs un emploi s'avèrent en revanche plus souvent expérimentateurs et usagers dans l'année. En cela ils se distinguent nettement des élèves et étudiants qui ne financent pas leurs études.

Globalement, toutefois, les étudiants du supérieur, contrairement à une idée répandue, ne se montrent pas plus souvent consommateurs que les actifs occupés du même âge (Beck et al., 2007). Enfin, les chômeurs affichent pour leur part des niveaux d'usage plus élevés. 
Tableau 3 : Odds ratios ajustés $(O R)^{\star}$ pour le statut scolaire et professionnel en fonction des différents usages de cannabis parmi les 15-34 ans

\begin{tabular}{|r|c|c|c|c|}
\hline & expérimentation & $\begin{array}{c}\text { usage au cours } \\
\text { de l'année }\end{array}$ & $\begin{array}{c}\text { usage au } \\
\text { cours du } \\
\text { mois }\end{array}$ & $\begin{array}{c}\text { usage } \\
\text { régulier }\end{array}$ \\
\hline & OR & OR & OR & OR \\
\hline $\begin{array}{r}\text { étudiant } \\
\text { travaille+études }\end{array}$ & $\mathbf{0 , 8}$ & 1,0 & 1,0 & 0,8 \\
chômage & $\mathbf{1 , 2}$ & $\mathbf{1 , 3}$ & 1,1 & 1,2 \\
inactifs & 1,1 & $\mathbf{1 , 3}$ & $\mathbf{1 , 4}$ & $\mathbf{1 , 6}$ \\
actifs occupés : référence & 0,8 & 1,0 & 1,0 & 1,4 \\
\hline
\end{tabular}

* Sur l'âge, le sexe, la vie de couple, les enfants, le niveau de diplôme, la taille de l'unité urbaine de résidence et l'équipement téléphonique (liste blanche/orange, rouge, mobile exclusif). En gras figurent les OR dont l'intervalle de confiance à $95 \%$ ne contient pas 1 ; ceux en italiques sont significativement inférieurs à 1 . Un OR gras supérieur à 1 indique une surconsommation relative par rapport à la catégorie de référence pour l'indicateur considéré ; un OR gras inférieur à 1 indique une sous-consommation relative.

Source : Baromètre santé 2005, Inpes, exploitation OFDT

Le niveau de formation scolaire est également un facteur de clivage important. Ainsi, toutes choses égales par ailleurs, les modèles logistiques précédents permettent de montrer que la possession d'un diplôme de niveau Bac est globalement un facteur associé à l'expérimentation, comme à l'usage dans l'année ou le mois. En revanche, elle est associée à une diminution de l'usage régulier de cannabis : la proportion d'usagers réguliers parmi les possesseurs d'un diplôme du supérieur Bac +5 est ainsi presque inférieure de moitié à celle mesurée parmi ceux qui se sont arrêtés au Bac.

Ce dernier point pourrait s'expliquer par une perception accrue des dangers du produit ou une attention supérieure portée à sa santé, avec l'élévation du niveau d'instruction. Il s'agirait alors d'un résultat comparable à celui retrouvé pour le tabac, moins souvent fumé parmi les personnes dont la position sociale est élevée. La raison de ce comportement pourrait toutefois également résider dans l'influence d'autres facteurs sociaux liés à l'instruction : type de sociabilité et d'environnement familial, contraintes ou investissement professionnels plus importants, etc.

\section{Mais des différences faibles entre les catégories d'actifs occupés}

Parmi les actifs occupés âgés de 15 à 34 ans (génération qui concentre l'essentiel des usagers de cannabis, surtout fréquents), les différences entre PCS se révèlent faibles, comme le laissait entrevoir la figure 2. Toutes choses égales par ailleurs, il persiste des écarts pour l'expérimentation avec notamment une surconsommation des cadres, des artisans et commerçants et enfin des professions intermédiaires comparativement aux ouvriers et, à l'inverse, une sous-expérimentation des agriculteurs exploitants.

Pour les usages plus récents, les différences s'estompent mais les cadres apparaissent néanmoins moins souvent consommateurs réguliers que les ouvriers, ce qui va dans le sens des résultats observés pour le niveau de diplôme rappelés précédemment. Ainsi, le cannabis est bien diffusé de manière relativement homogène dans le monde du travail. Ces résultats restent évidemment très grossiers, vu l'hétérogénéité des situations regroupées au sein d'une PCS donnée. 


\section{Un regard sur l'adolescence}

En 2005, l'enquête Escapad a permis l'analyse des réponses de plus de 29000 adolescents français de 17 ans. L'existence de ces données très précises invite à décrire à part la situation des adolescents, catégorie très sensible d'usagers en raison de l'ampleur de la diffusion des usages en leur sein, mais aussi du lien existant entre précocité d'usage et usage problématique.

\section{Les garçons nettement surconsommateurs}

À l'adolescence, comme dans la population adulte, le cannabis est de très loin la principale drogue illicite consommée. En 2005, 49,5\% des jeunes français de 17 ans déclaraient avoir déjà consommé du cannabis au cours de leur vie, $27,9 \%$ en avoir pris au cours des trente derniers jours, 10,8 \% en avoir pris de façon régulière et $5,2 \%$ en avoir pris quotidiennement au cours de cette période (Beck et al., 2006b). La proportion de garçons augmente avec l'élévation de la fréquence d'usage considérée : le sex ratio vaut 1,2 pour l'expérimentation et l'usage au cours de l'année, 1,5 pour l'usage au cours du mois, 2,4 pour l'usage régulier et 2,5 pour l'usage quotidien. Il s'agit aussi de la substances psychoactive illicite dont l'expérimentation est la plus précoce (vers 15 ans en moyenne, lorsqu'elle est interrogée à 17 ans. L'expérimentation a lieu très massivement au cours de l'adolescence, les prévalences croissant très rapidement avec l'âge au cours de cette période de la vie.

\section{Des usages liés à la situation scolaire, à la sociabilité mais moins au milieu social}

Comme au sein de la population adulte, certaines caractéristiques sociales (scolaires et familiales) permettent de différencier les comportements de consommation. Ainsi les usages s'avèrent plus répandus parmi les jeunes qui se sont orientés vers une filière scolaire courte ou professionnelle, et davantage encore parmi les jeunes sortis du système scolaire (tableau 4) et le même résultat est observé pour le redoublement au cours de la scolarité. La relégation scolaire semble ainsi constituer un facteur favorisant la consommation. Le milieu social familial joue également un rôle, mais dans une moindre mesure :

l'expérimentation apparaît certes plus fréquente avec l'élévation de la profession et catégorie sociale des parents, mais les usages plus fréquents, comme l'usage régulier, sont tout à fait similaires dans ces segments de populations (tableau 5).

À l'instar de ce qui a été présenté en population adulte, ces résultats peuvent être testés dans une analyse logistique contrôlant le sexe, le milieu social, la situation scolaire et le redoublement, l'union parentale et la décohabitation de l'adolescent (ce qui est parfois le cas, pour des raisons d'éloignement du foyer familial de l'école ou de l'entreprise). Le lien entre scolarité et usage apparaît alors maintenu, les jeunes ayant redoublé, ceux en situation de relégation ou de sortie du système scolaire présentent des fréquences d'usage nettement supérieures que leurs homologues scolarisés au parcours plus classique.

Parallèlement, les fréquences d'usage, de l'expérimentation à l'usage régulier apparaissent nettement diminuées parmi les jeunes issus de familles les moins favorisées sur le plan social et économique (tableau 6).

Notons que d'autres facteurs, comme le fait de vivre au sein de foyer parental ou l'union des parents jouent également un rôle, notamment en raison des possibilités de surveillance formelle ou informelle que ces situations autorisent. 
Ainsi, toutes choses égales par ailleurs, dans l'analyse modélisant l'usage régulier, la séparation des parents (divorce ou autre cause) est associée à un OR de 1,7, autrement dit à un usage nettement plus répandu, relativement aux jeunes dont les deux parents sont unis, un OR similaire étant observé pour la décohabitation de l'adolescent.

Ces résultats contredisent ainsi l'idée répandue que le cannabis, en tant que drogue illicite, serait plus consommé dans les milieux populaires ou défavorisés : dès l'adolescence, il se trouve répandu dans tous les milieux, mais à parcours scolaire et situations familiales comparables, il apparaît plus fréquent au sein des milieux économiquement favorisés.

Ces enseignements occultent néanmoins une dimension très importante de l'usage de cannabis, le plaisir et la convivialité. En effet, l'intensité de la sociabilité et des contacts amicaux clive les usages encore bien plus nettement que le parcours scolaire ou le capital socio-économique des parents tel que déclaré par l'enfant : la proportion d'usagers de cannabis au cours du mois triple avec la fréquence des sorties dans les bars (d'aucune sortie au cours des douze derniers mois à des sorties presque quotidiennes) et elle est même multipliée par six avec les fréquences des soirées chez soi ou chez des amis (tableau 7).

Tableau 4 : usages de cannabis suivant le statut scolaire et professionnel à 17 ans ( \%)

\begin{tabular}{|c|c|c|c|}
\hline & expérimentation & usage au cours du mois & usage régulier \\
\hline élève, étudiant & 47,8 & 26,0 & 9,0 \\
\hline $\begin{array}{l}\text { apprentissage, } \\
\text { formation alternée }\end{array}$ & 57,1 & 37,2 & 18,1 \\
\hline $\begin{array}{r}\text { actif (chômeur ou } \\
\text { occupé) }\end{array}$ & $60,1^{* \star *}$ & $41,5^{\star \star \star}$ & $27,0^{\star * *}$ \\
\hline
\end{tabular}

Exemple de lecture : 47,8\% des élèves et étudiants de 17 ans sont expérimentateurs de cannabis, $26.0 \%$ usagers au cours du mois et $9,0 \%$ usagers réguliers. ${ }^{* * *}$ : test du $\mathrm{Chi}^{2}$ significatif au seuil 0,001 .

Source : Escapad 2005, OFDT

Tableau 5 : usages de cannabis suivant le milieu social familial déclaré à 17 ans (\%)

\begin{tabular}{|c|c|c|c|}
\hline milieu social" & expérimentation & usage au cours du mois & usage régulier \\
\hline " défavorisé » & 43,1 & 26,1 & 10,4 \\
" modeste » & 46,3 & 26,2 & 10,5 \\
« moyen » & 49,5 & 28,0 & 11,0 \\
« favorisé » & 53,5 & 29,6 & 10,5 \\
" très favorisé 》 & $56,2^{* \star *}$ & $31,3^{* * *}$ & $11,1 \mathrm{NS}$ \\
\hline
\end{tabular}

Exemple de lecture : 43,1\% des adolescents de 17 ans dont la famille est classée dans la catégorie « défavorisée » (voir ci-dessous) disent avoir expérimenté le cannabis. ${ }^{* \star *}$ et NS : test du Chi ${ }^{2}$ significatif au seuil 0,001 et non significatif au seuil 0,05 .

Source : Escapad 2005, OFDT

* Évalué par la Profession et catégorie sociale (Pcs) la plus élevée du couple des parents, parmi 11 choix assortis d'exemples de professions, selon la répartition suivante : " défavorisé » indique que les deux parents sont déclarés inoccupés par l'enfant ; " modeste » qu'ils sont au maximum ouvrier ou employé ; « moyen » qu'ils sont au plus une profession intermédiaire ; « favorisé » que l'un seulement des parents est cadre, chef d'entreprise, artisan ou commerçant ; " très favorisé " que les deux le sont. Ces catégories recoupent celles de l'Insee, mais ne sont pas identiques. 
Tableau 6 : OR ajustés pour le milieu social relativement à quelques usages de cannabis à 17 ans (\%)

\begin{tabular}{|r|c|c|c|}
\hline milieu social" & expérimentation & usage au cours du mois & usage régulier \\
\hline " défavorisé » & 0,5 & 0,6 & 0,6 \\
«modeste " & 0,6 & 0,7 & 0,7 \\
«moyen » & 0,7 & 0,8 & 0,9 \\
" favorisé " & 0,8 & 0,9 & 0,8 \\
" très favorisé " & $-1-$ & $-1-$ & $-1-$ \\
\hline
\end{tabular}

Les variables d'ajustement sont : le sexe, la situation scolaire présente (scolarisation classique, apprentissage ou formation alternée, sortie du système scolaire), le redoublement au cours de la scolarité (aucun, un, deux ou plus), l'union des parents, la vie hors foyer du répondant et la Pcs du couple des parents telle que déclarée par l'enfant. En gras figurent les OR dont l'intervalle de confiance à $95 \%$ ne contient pas 1 ; ceux en italiques sont significativement inférieurs à 1 . Un OR gras inférieur à 1 indique une sous-consommation relative.

Tableau 7 : usages de cannabis suivant la sociabilité à 17 ans (\%)

\begin{tabular}{|c|c|c|c|c|c|}
\hline $\begin{array}{c}\text { sortie dans les cafés, bars, } \\
\text { pubs }\end{array}$ & $\begin{array}{c}\text { usage au } \\
\text { cours du } \\
\text { mois }\end{array}$ & $\begin{array}{c}\text { usage } \\
\text { régulier }\end{array}$ & $\begin{array}{c}\text { en soirée chez des amis } \\
\text { ou chez soi }\end{array}$ & $\begin{array}{c}\text { usage au } \\
\text { cours du } \\
\text { mois }\end{array}$ & $\begin{array}{c}\text { usage } \\
\text { régulier }\end{array}$ \\
\hline jamais & 14,6 & 6,6 & jamais & 7,8 & 2,1 \\
moins d'une fois par mois & 19,5 & 6,4 & moins d'une fois par mois & 11,1 & 2,4 \\
une ou deux fois par mois & 30,2 & 10,4 & une ou deux fois par mois & 23,0 & 4,9 \\
au moins une fois par & 39,8 & 15,7 & au moins une fois par & 40,0 & 16,8 \\
$\begin{array}{c}\text { semaine } \\
\text { tous les jours ou presque }\end{array}$ & $44,2^{* * *}$ & $19,1^{\star * *}$ & tous les jours ou presque & $52,3^{\star * *}$ & $31,3^{\star * *}$ \\
\hline
\end{tabular}

Exemple de lecture : 14,6\% des adolescents de 17 ans déclarant n'être jamais allé dans un café, bar ou pub avec des amis au cours des douze derniers mois disent avoir fumé du cannabis dans les trente derniers jours. ${ }^{* * *}$ : test du Chi ${ }^{2}$ significatif au seuil 0,001 .

Source : Escapad 2005, OFDT

Au final, les usages de cannabis sont répandus dans tous les segments de la population et sur tout le territoire national. Le genre et l'âge restent les principaux déterminants démographiques, mais le niveau d'instruction scolaire, l'activité professionnelle et le type d'emploi ou encore la zone de résidence apparaissent également discriminants. Ainsi, les usages de cannabis paraissent plus fréquents parmi les hommes et les jeunes générations, mais aussi, par exemple, parmi les chômeurs, tandis que les différences entre les actifs occupés s'avèrent plus faibles. De même, l'expérimentation et les usages occasionnels se révèlent plus répandus parmi les personnes diplômées du supérieur, au contraire des usages plus réguliers. Ce résultat se retrouve pour la PCS. Ces déterminants sociaux, dont certains sont construits a posteriori, permettent de décrire et de rendre compte des usages dans la population mais ne peuvent suffire à les expliquer : souvent pluridimensionnels, ils traduisent et résument tout en les masquant partiellement, les opportunités, les choix et les préférences individuelles de consommation de produits psychoactifs. Ils occultent en particulier que les usages sont très nettement liés à une sociabilité et à un mode de vie. Comme le soulignent les usagers lorsqu'ils énoncent leurs motifs de consommation et d'abandon, le cannabis est partie intégrante de la vie de nombreuses personnes, qui ne semblent pas en éprouver de difficultés majeures. 


\section{L'intensité et la fréquence des consommations}

L'intensité de la consommation peut être approchée par le nombre de joints fumés lors de la dernière consommation. Près de la moitié (44\%) des consommateurs réguliers de cannabis de 18 ans ou plus ont « fumé la dernière fois » trois joints ou plus. À 17 ans, les consommateurs réguliers, mais non quotidiens, de cannabis sont relativement deux fois moins nombreux que les consommateurs quotidiens à avoir consommé 5 joints ou plus la dernière fois.

Le nombre de joints fumés la dernière fois varie fortement avec la fréquence d'usage : alors que $13 \%$ seulement des usagers « non réguliers » (c'est à dire ayant déclaré avoir fumé au cours des sept jours précédant l'enquête, mais moins de dix fois au cours des trente derniers jours) disent avoir fumé au moins trois joints lors de leur dernier jour de consommation, cette proportion atteint $43 \%$ parmi les fumeurs réguliers ( $\mathrm{p}<0,001)$. Quel que soit le jour examiné, l'intensité de la consommation augmente avec la fréquence d'usage : par exemple, $42 \%$ des fumeurs réguliers déclarent avoir fumé au moins trois joints le samedi précédant l'enquête, contre $12 \%$ parmi les fumeurs actuels.

\section{Les motifs de consommation et d'arrêt}

Parmi les personnes âgées de 15 à 64 ans ayant déjà consommé du cannabis mais ne l'ayant pas fait au cours des douze derniers mois, une nette majorité donne comme raison principale de l'arrêt de leur consommation le désintérêt $(78,8 \%)$. Les autres raisons invoquées s'avèrent nettement moins fréquentes : les principales sont le fait de ne pas avoir aimé la première fois $(4,2 \%)$, l'absence d'opportunité $(3,9 \%)$, la peur pour la santé $(3,9 \%)$, un dégoût des drogues dans leur ensemble $(2,6 \%)$, le fait de ne plus ressentir de plaisir $(2,3 \%)$, de se trouver trop vieux $(1,9 \%)$, la peur de la dépendance $(1,4 \%)$, ou enfin le décalage par rapport à son milieu social $(0,9 \%)$.

Les motivations des individus ayant consommé au cours de l'année apparaissent quant à elles plus contrastées. Les principales raisons sont la curiosité $(21,9 \%)$, la recherche de la détente $(19,9 \%)$, l'envie de goûter $(13,9 \%)$, d'essayer $(11,0 \%)$. Le pouvoir de socialisation attribué au cannabis permet de distinguer un deuxième groupe de raisons invoquées : l'envie de s'amuser $(10,3 \%)$, la complicité avec d'autres personnes $(8,9 \%)$, la conformité (« Pour faire comme tout le monde $»: 8,4 \%$ ), la convivialité $(7,5 \%)$. Notons que 7,2\% disent avoir recherché le plaisir, 2,7\% l'ivresse, $2,2 \%$ avoir fumé pour oublier leurs problèmes, $1,6 \%$ pour être défoncé et $1,1 \%$ pour trouver le sommeil. Très peu d'anciens consommateurs disent avoir abandonné à cause de problèmes rencontrés lors de leur usage. L'usage de cannabis est donc surtout motivé par des considérations hédoniques, et son abandon l'est tout autant par la lassitude ou le manque d'intérêt. Néanmoins, il convient de s'attarder sur les usagers en situation d'usage problématique. 


\section{La surconsommation et l'usage problématique de cannabis}

\section{Pourquoi définir un seuil d'usage problématique}

Si une très grande majorité des usagers de cannabis ne semblent pas s'exposer à des risques majeurs pour leur santé, les conséquences sanitaires de l'extension de son usage (hors pathologies analogues à celles occasionnées par la consommation de tabac) ne sont pas encore claires (Inserm, 2001). Cependant, on assiste partout en Europe à une demande croissante de soins et de prises en charges médicales de la part d'usagers de cannabis (OEDT, 2003). Celle-ci souligne l'importance de la question de la définition d'un seuil au-delà duquel il serait légitime de considérer que l'usage est problématique ou dangereux. La problématique n'est pas nouvelle, mais elle est ressurgit néanmoins actuellement de manière criante, ce qui suggère, au vu de l'ancienneté et de l'ampleur de l'usage de cannabis dans nos sociétés, que son observation n'est pas en elle-même une condition suffisante de la recherche dans ce domaine. D'autres facteurs doivent entrer en jeu pour le constituer comme problème de santé publique.

À l'instar de ce qui est fait dans les principaux pays européens, les enquêtes en population générale ou scolaire menées auprès des jeunes depuis quelques années en France ne contiennent pas de données concernant la dépendance, l'usage nocif (selon la typologie retenue dans la CiM-10 ${ }^{7}$ ) ou l'abus (selon les critères du DSM$\mathrm{IV}^{8}$ ) de ces substances, qui peuvent être considérées comme des usages immédiatement ou rapidement dommageables pour l'individu. La surconsommation est, au sein de ces enquêtes, définie en termes purement quantitatifs comme un niveau d'usage qui se distinguerait d'une norme définie comme une consommation modérée. Depuis peu, un certain nombre d'équipes travaillent à construire des outils de repérage de l'usage problématique de cannabis, en s'appuyant notamment sur des travaux de validation épidémiologique et clinique engagés en France (Karila et al., 2004) et à l'étranger (Adamson et Sellman, 2003 ; Dale, 2003 ; Heishman et al., 2001 ; Okulicz-Kozaryn et Sieroslawski, 2004 ; Martin et al., 2006) ainsi que sur un travail de recension des expériences internationales sur cette question (Beck et Legleye, 2007).

\section{Des difficultés conceptuelles}

L'abus et l'usage nocif caractérisent des usages immédiatement dommageables pour l'individu. Ils font partie d'un continuum d'usages qui va de l'abstinence à la dépendance qui peut être en elle-même considérée comme un usage nocif. L'abus peut englober des problèmes légaux (par exemple les interpellations, arrestations et condamnations liées à l'usage), et des comportements à risques (comme des usages augmentant les risques physiques liés à la pratique d'une activité de loisir ou professionnelle) qui ne sont pas inclus dans la définition de l'usage nocif. En dépit de ces différences, ces définitions ne permettent pas d'ordonner ces usages de façon univoque selon une gravité croissante, parce qu'elles présentent des aspects communs, à l'instar des reproches de la part de la famille ou des proches, ou les difficultés personnelles ou sociales découlant de l'usage. En sus, ces concepts n'excluent pas la dépendance stricto sensu.

Deux autres concepts sont parfois utilisés, concepts qui sont des composantes de la dépendance selon le DSM-IV : les dépendances physique et psychologique.

\footnotetext{
${ }^{7}$ Classification internationale des maladies

${ }^{8}$ Diagnostic and Statistical Manual of Mental Disorders $-4^{\mathrm{e}}$ édition
} 
Le premier comprend la tolérance et les syndromes de manque. Ces concepts sont souvent présents dans les tests de repérage des usages problématiques, qu'ils soient spécifiques au cannabis ou non.

Dans une perspective résolument non psychopathologique, certaines définitions de l'usage problématique de drogues sont purement pragmatiques. C'est le cas de la considération de certains usages de certaines drogues ou de certains modes d'usages en particulier (OEDT, 2003), comme l'injection d'opiacés, les usages réguliers de substances illicites autres que le cannabis. Mais une telle approche convient mal au cas du cannabis en raison de la fréquence de son usage dans la population, et du peu de risques immédiats qu'il fait courir à l'usager. Dans les enquêtes en population générale, l'usage problématique de cannabis est souvent caractérisé par une intensité d'usage arbitraire, définie en fréquence de consommation ou en nombre de joints fumés (Simon, 2004). Cette notion est fondée sur l'idée raisonnable que les risques pour l'individu, quels qu'ils soient, sont plus grands à mesure qu'il consomme davantage. Les seuils utilisés sont cependant arbitraires et les études épidémiologiques montrent que les liens statistiques entre problèmes et usage apparaissent dès les plus bas niveaux mesurables (Ramström, 2003), et qu'ils doivent être interprétés avec précaution suivant le contexte sociodémographique, etc.

Les objectifs et la portée des concepts utilisés en addictologie, en théorie comme en pratique, varient donc, depuis la description d'états non problématiques en soi mais évoquant un tableau précurseur d'une situation susceptible de se dégrader, jusqu'au critère de dommage avéré et finalement la dépendance. Leur sens, leur usage et leur utilité sont donc conditionnés à leur articulation avec le système de soin et de prise en charge mais aussi en dernier ressort avec le système répressif. En retour, cette articulation souligne la difficulté de parvenir à une quantification du risque d'aggravation des usages les moins immédiatement dommageables pour l'individu, et donc en définitive de repérer à l'avance les problèmes futurs et les dangers à moyen et long terme. Faut-il se restreindre aux dommages de santé physique ou mentale, ou bien encore aux problèmes d'ordre social ou familial rencontrés par l'usager, ou bien faut-il inclure les problèmes causés par l'individu à la société ? La dépendance est-elle un problème en soi ? Le niveau d'usage observé dans le pays, les contextes économique, moral, ainsi que l'articulation des systèmes sanitaires et répressifs ont un impact considérable sur ces questions. Les concepts et les critères du DSM ou de la CIM (abus, usage nocif et dépendance) sont une première approche mais leur universalité et leur pérennité sont aujourd'hui clairement mises en cause dans les discussions engagées autour de la mise au point de la version 5 du DSM.

\section{Le délicat passage du concept au test de repérage}

Le repérage consiste, une fois le concept défini et choisi, à isoler les usages répondant aux critères du concept. Encore faut il réussir à traduire le concept en critères, puis questions susceptibles d'être posées dans une enquête ou posées par un spécialiste à son patient, ce qui ne peut être fait de façon univoque. Comme être sûr que la liste des critères utilisés est la bonne ? Que les questions opérationnalisent correctement les critères ? Combien de critères faut-il retenir? De plus, les concepts doivent possiblement être adaptés à la population ciblée : par exemple, la tolérance et le manque évoqués dans le DSM-IV sont, pour certains auteurs relativement inadaptés à la description des usages problématiques de cannabis chez les jeunes (Harrison et al, 1998 ; Buckstein et Kaminer, 1994). Les 
tests développés pour les adolescents présentent ainsi souvent des écarts importants comparés aux tests adaptés aux adultes (Inserm, 2001).

\section{Validation mesure de la qualité}

La validation d'un test de repérage est cumulative et circulaire. Elle repose en premier lieu sur la comparaison du test candidat à un test de référence que l'on supposera fournir une traduction exacte du concept visé, traduction dont il est par définition impossible de s'assurer de l'exactitude. Dans un second temps, une confirmation de la qualité du test en situation clinique peut voir le jour, lorsque son résultat est confronté à un diagnostic médical. Le diagnostic médical, censé être la référence, repose sur les mêmes concepts de base que le test de référence, mais y adjoint un regard clinique et un jugement subjectif sur la gravité de la situation du patient. Faut-il dès lors se fonder sur le test de référence ou le jugement du clinicien ? En fait, l'évaluation de la qualité du test se fait par l'examen d'indicateurs épidémiologiques (sensibilité, spécificité, etc.) mais aussi par l'accroissement de la confiance dans le test résultant de la diffusion de son utilisation à l'échelle la plus vaste possible. La qualité du test se mesure en partie à son utilité, qui peut être grande malgré les défauts connus. Elle est ainsi dans une certaine mesure contingente, culturelle et historique. Le test pourra en effet être critiqué en regard d'un changement de paradigme concernant l'approche conceptuelle (événement relativement courant comme le montre l'histoire de l'addictologie), ou d'un changement de la position des drogues et de ses usagers dans la société, bref, lorsque ses limites cliniques et pratiques deviendront trop gênantes en regard des bénéfices qu'il apporte.

Au final, le travail peut encore se compliquer lorsqu'il s'agit de définir un test valable dans une majorité de pays, de cultures et de pratiques de consommations. Précisons de plus que le repérage n'est pas une fin en soi : il peut être mené dans des enquêtes de grande échelle afin d'obtenir une mesure valable sur l'ensemble de la population et alimenter des recherches sociologiques ou épidémiologiques, soit servir à un premier criblage systématique lors des consultations de médecine générale précédant des entretiens plus approfondis. En cela, il doit être articulé à un projet de recherche ou une prise en charge et un retour d'information en direction du patient ; ce qui ne manque pas de questionner les épidémiologistes et concepteurs d'enquêtes en population générale. Le repérage est en effet une opération de classement dont les implications médicales, sociales voire légales ne sont pas neutres et qui comporte de ce fait une dimension politique évidente.

\section{L'expérience française}

En France, les premières explorations épidémiologiques concernant l'usage de cannabis ont été menées au début des années 2000 , montrant que des contextes a priori non festifs comme le fait de fumer seul ou avant midi semblent trop peu spécifiques : ils peuvent couvrir des situations où l'usage de cannabis est maîtrisé et n'apparaît pas associé à un quelconque mal-être ou à des difficultés (Beck et $a l ., 2000)$. En 2001, une autre analyse a permis de démontrer que si la santé mentale des usagers réguliers de cannabis apparaît moins bonne que celle des autres individus enquêtés, le lien tend à disparaître lorsqu'on observe simultanément d'autres variables liées au mode de vie (contexte familial, intensité de la sociabilité, violences subies...) ou encore les usages d'alcool ou de tabac (Peretti-Watel et al., 2002). 
La plupart des tests existant dans la littérature s'avèrent relativement longs, complexes, ou visent essentiellement à repérer des usages rares, très intensifs et dommageables pour l'individu à partir des critères utilisés dans le DSM ou la CIM pour les opiacés ou l'alcool (Beck et Legleye, 2007). C'est pourquoi a été développé le questionnaire CAST (Cannabis Abuse Screening Test) en 2002 à partir de la littérature existante (Reynaud et al., 2002). Il n'existe pas en effet, à notre connaissance, d'instruments en langue française spécifiques au cannabis, à l'exception du questionnaire québécois Depado (Landry et al., 1992) qui traite simultanément de toutes les substances illicites et du MiNI (Lecrubier et al., 1997 ; Sheehan et al., 1998) qui repère la dépendance ou l'abus à partir des critères du DSM. Le CAST a été testé et validé dans une enquête en 2003 auprès de 1728 élèves et étudiants (enquête Adotecno, adolescents, techniques d'évaluation des consommations nocives), (Legleye et al., 2007). Cette validation a été opérée par rapport au questionnaire Posit (Problem oriented screening interview for teenagers), développé aux États-Unis par le NIDA (National Institute for Drug Abuse). (Rahdert, 1991 ; Knight et al., 2001). Le test CAST est le suivant :

Au cours des 12 derniers mois :

\begin{tabular}{|c|c|c|c|c|c|}
\hline Une seule croix par ligne & Jamais & Rarement & $\begin{array}{l}\text { De temps en } \\
\text { temps }\end{array}$ & $\begin{array}{l}\text { Assez } \\
\text { souvent }\end{array}$ & $\begin{array}{l}\text { Très } \\
\text { souvent }\end{array}$ \\
\hline Avez-vous fumé du cannabis avant midi ? & ${ }_{1} \square$ & $2 \square$ & ${ }_{3} \square$ & $4 \square$ & $5 \square$ \\
\hline $\begin{array}{l}\text { Avez-vous fumé du cannabis lorsque vous étiez } \\
\text { seul(e)? }\end{array}$ & ${ }_{1} \square$ & $2 \square$ & ${ }_{3} \square$ & ${ }_{4} \square$ & $5 \square$ \\
\hline $\begin{array}{l}\text { Avez-vous eu des problèmes de mémoire quand } \\
\text { vous fumez du cannabis? }\end{array}$ & ${ }_{1} \square$ & $2 \square$ & ${ }_{3} \square$ & ${ }_{4} \square$ & $5 \square$ \\
\hline $\begin{array}{l}\text { Des amis ou des membres de votre famille vous } \\
\text { ont ils dit que vous devriez réduire votre } \\
\text { consommation de cannabis? }\end{array}$ & ${ }_{1} \square$ & $2 \square$ & ${ }_{3} \square$ & ${ }_{4} \square$ & $5 \square$ \\
\hline $\begin{array}{l}\text { Avez-vous essayé de réduire ou d'arrêter votre } \\
\text { consommation de cannabis sans y arriver? }\end{array}$ & ${ }_{1} \square$ & $2 \square$ & ${ }_{3} \square$ & ${ }_{4} \square$ & $5 \square$ \\
\hline $\begin{array}{l}\text { Avez-vous eu des problèmes à cause de votre } \\
\text { consommation de cannabis (dispute, bagarre, } \\
\text { accident, mauvais résultat à l'école...) ? } \\
\text { Lesquels : /.................................... }\end{array}$ & ${ }_{1} \square$ & $2 \square$ & ${ }_{3} \square$ & ${ }_{4} \square$ & $5 \square$ \\
\hline
\end{tabular}

\section{Validation et utilisation}

L'enquête a permis d'établir que le CAST possède de bonnes caractéristiques psychométriques et statistiques. (Legleye et al., 2007). En pratique, le score est calculé de la façon suivante : les deux premières questions cotent 1 à partir de la réponse " de temps en temps », 0 sinon ; les quatre suivantes cotent 1 dès la modalité « rarement ». Un score supérieur ou égal à 4 suggère fortement un usage problématique. Par rapport au facteur A du POSIT qui repère les abus de produits psychoactifs au sens du DSM-IV, le CAST ainsi étalonné présente une sensibilité de $93 \%$ et une spécificité de $81 \%$. Sa qualité réside dans sa brièveté et la très grande facilité à y répondre. L'étude Adotecno a en effet permis de montrer que le taux de réponse au CAST est nettement supérieur à celui enregistré pour les tests concurrents CRAFFT (en quatre questions) et ALAC (en 11 questions), qui 
contiennent uniquement des questions aux réponses binaires (oui/non) (ALAC, 1996).

Dans l'enquête Escapad 2003, la proportion de jeunes de 17-18 ans ainsi repérés atteint $8,9 \%(12,4 \%$ des garçons pour $4,4 \%$ des filles $)$, ce qui représente $53 \%$ des usagers réguliers et $69 \%$ des usagers quotidiens. Ce point souligne clairement que le simple recueil de la fréquence de consommation n'assure pas à lui seul une mesure correcte, dans toutes les situations, des problèmes liés à l'usage.

\section{Un usage problématique lié à la précocité de l'expérimentation}

La précocité du premier usage est un facteur prédictif important de l'intensité de l'usage à l'adolescence : alors que parmi les adolescents de 17-18 ans déclarant avoir fumé leur premier joint à 16 ans, $4 \%$ sont usagers quotidiens de cannabis et $11 \%$ usagers réguliers non quotidiens, ces proportions atteignent $18 \%$ et $22 \%$ parmi ceux ayant expérimenté à 14 ans et $39 \%$ et $25 \%$ parmi ceux ayant expérimenté à 12 ans. La proportion d'usagers problématiques selon le CAST suit la même progression avec la précocité : elle vaut $9 \%$ parmi les adolescents de 17 18 ans ayant fumé leur premier joint à 16 ans, $29 \%$ parmi ceux l'ayant fait à 14 ans et $50 \%$ parmi ceux qui l'ont fait à 12 ans.

Si ce résultat est important en situation clinique où il est crucial de connaître avec précision l'usage d'un patient afin de juger de sa gravité, il n'en reste pas moins d'une interprétation délicate. L'usage problématique est une mesure de l'usage actuel éventuellement prédictive de l'évolution de l'usage futur. La précocité de l'expérimentation fournit quant à elle un indicateur de l'ancienneté de la consommation. Ainsi, en face de deux usagers de mêmes âges, conditions sociales, etc. ayant les mêmes usages actuels de cannabis, il est difficile d'affirmer que celui qui a fumé le plus précocement son premier joint présente davantage de signes de gravité que l'autre. Si le lien entre précocité et gravité existe, il est même possible de considérer que l'usager le plus ancien présente les signes les plus nombreux de stabilité de son usage au cours du temps. Il s'avère dès lors important de chercher à disposer d'indications plus précises sur la dynamique d'entrée dans les consommations.

\section{Conclusion}

Si l'épidémiologie de la surconsommation de cannabis en est à ses balbutiements, elle a déjà donné quelques résultats importants qui rejoignent la pratique des acteurs de terrain, tel que le lien fort entre une telle pratique et une entrée précoce dans les usages. Les véritables avancées sont toutefois à venir, lorsque les instruments de mesure de l'usage problématique de cannabis auront été validés tant au niveau épidémiologique qu'en clinique et utilisés dans les enquêtes en population générale. Ces progrès pourraient accompagner utilement les actions de repérage précoce et d'interventions brèves menées autour du cannabis, actions qui ont notamment montré leur efficacité sur les pratiques d'alcoolisation. 


\section{Bibliographie}

Adamson SJ, Sellman JD : “A prototype screening instrument for cannabis use disorder: the Cannabis Use Disorders Identification Test (CUDIT) in an alcohol-dependent clinical sample" - In Drug and Alcohol Review 22 : 309315 (2003)

ALAC : Guidelines for Alcohol and Drug Assessment: A review of alcohol and drug screening, diagnostic and evaluation instruments - Wellington, Alcohol Advisory Council of New Zealand (1996)

Beck F : Entre représentativité des échantillons et représentation des usages : l'apport des enquêtes en population générale à la compréhension des usages de drogues, Thèse de sociologie, Université René Descartes Paris V (2006)

Beck F, Legleye S : "Measuring cannabis related problems and dependence at the population level" - In Room R, Rödner S (dir.): Cannabis monograph, European Monitoring Center on Drug and Drug Addiction, EMCDDA (2007)

Beck F, Legleye S, Spilka S, Briffault X, Gautier A, Lamboy B, Leon C, Wilquin J-L : « Les niveaux d'usage des drogues en France en 2005, exploitation des données du Baromètre santé 2005 »-In Tendances 48 1-6 (2006a)

Beck F, Legleye S, Spilka $\mathrm{S}$ : « Les drogues à 17 ans, évolutions, contextes d'usage et prises de risque $»-$ In Tendances, $\mathrm{n}^{\circ} 49$ (2006b)

Beck F, Legleye S, Peretti-Watel P : Regards sur la fin de l'adolescence : consommations de substances psychoactives lors de l'enquête Escapad 2000 Paris, OFDT (2000)

Dale, A : "A marijuana screening inventory (experimental version): description and preliminary psychometric properties" - In American Journal of Drug and Alcohol Abuse, volume 29( 3) : 619-646 (2003)

Heishman SJ, Singleton EG, Liguori : "A Marijuana craving questionnaire: development and initial validation of a self-report instrument" - In Addiction 96 (7) : 1023-1034 (2001)

Inserm : Cannabis, quels effets sur le comportement et la santé ? - Inserm Expertise collective (2001)

Karila L, Legleye S, Donnadieu S, Beck F, Corruble E, Reynaud M : «Consommations nocives de produits psychoactifs; résultats préliminaires de l'étude Adotecno » - In Alcoologie et Addictologie, volume 26 (2) : 99-109 (2004)

Knight Jr, Goodman E, Pulerwitz T et al. : "Reliability of the POSIT in adolescent medical practice" - In Journal of adolescent health 29 : 125-130 (2001)

Landry M, Ishak I, Vaugeois $\mathrm{P}$, Trépanier M : Les cahiers de recherche $d u$ RISQ, Montréal (1992). Sur internet à : www.risq-cirasst.umontreal.ca

Lecrubier Y, Sheehan D, Weiller E et al. : "The MINI International neuropsychiatric interview (MINI): a short diagnostic structured interview : reliability and validity according to the CIDI" - In European Psychiatry 12 : 224-131 (1997)

Legleye S, Karila L, Beck F, Reynaud M : « Validation of the CAST, a Cannabis Abuse Screening Test in general population »- In Journal of Substance Use, (soumis) 
Martin G, Copeland J, Gates P, Gilmour S : "The Severity of Dependence Scale (SDS) in an adolescent population of cannabis users: Reliability, validity and diagnostic cut-off' - In Drug and Alcohol Dependence 83 : 90-93 (2006)

OEDT : État du phénomène des drogues dans l'union européenne et en Norvège Lisbonne, OEDT (2003)

Okulicz-Kozaryn K, Sieroslawski J : The Development of Screening Tests for Adolescents' Drug-and Marihuana-Problem Use - Paper presented at KBS, Helsinki (2004)

Peretti-Watel P, Legleye S, Beck F : « Santé mentale et usage de cannabis à la fin de l'adolescence : une relation qui déborde le cadre pharmacologique »- In Médecine/Sciences 18, ${ }^{\circ}$ 4, $2002: 481-488$

Rahdert $\mathrm{E}:$ The adolescent assessment and referral system manual-DHHS Publication $n^{\circ}$ (ADM) 91-1735 - Rockville, MD, National Institute on Drug Abuse (1991)

Reynaud M : Usage nocif de substances psychoactives - Paris, La documentation française (2002)

Sheehan D, Lecrubier Y Sheehan KH, Amorim P, Janavs J, Weiler E, Hergueta T, Baker R, and Dunbar GC : "The Mini International Neuropsychiatric Interview (MINI), The development and validation of a structured diagnostic psychiatric interview for DSM-IV and ICD-10" - In Journal of Clinical Psychiatry 59 (20) : 22-33 (1998) 